\title{
Cognitive Protective Mechanism of Crocin Pretreatment in Rat Submitted to Acute High-Altitude Hypoxia Exposure
}

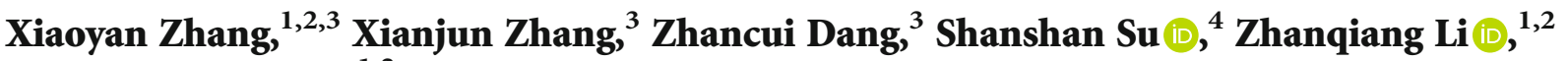 \\ and Dianxiang $\mathrm{Lu}(\mathrm{D})^{1,2}$ \\ ${ }^{1}$ Research Center for High Altitude Medicine, Key Laboratory of High Altitude Medicine, Ministry of Education, Qinghai University, \\ Xining 810001, China \\ ${ }^{2}$ Key Laboratory of Application and Foundation for High Altitude Medicine Research in Qinghai Province, Xining 810001, China \\ ${ }^{3}$ Department of Basic Medicine Science, Medical College, Qinghai University, Xining 810001, China \\ ${ }^{4}$ Technical Center of Xining Customs District, Key Laboratory of Food Safety Research in Qinghai Province, Xining 810003, China
}

Correspondence should be addressed to Zhanqiang Li; zhanqiang_li@163.com and Dianxiang Lu; ludianxiang@126.com

Received 18 March 2020; Revised 8 May 2020; Accepted 25 May 2020; Published 10 June 2020

Academic Editor: Jane Hanrahan

Copyright $\odot 2020$ Xiaoyan Zhang et al. This is an open access article distributed under the Creative Commons Attribution License, which permits unrestricted use, distribution, and reproduction in any medium, provided the original work is properly cited.

Inadequate oxygen availability at high altitude leads to oxidative stress, resulting in hippocampal neurodegeneration and memory impairment. In our previous study, we found that the cognitive dysfunction occurred when male SD rat was rapidly exposed to $4200 \mathrm{~m}$ of high altitude for 3 days. And we also found that crocin showed a cognitive protective effect under hypoxia by regulating SIRT1/PGC- $1 \alpha$ pathways in rat's hippocampus. In this article, focused on factors related to SIRT1/PGC- $1 \alpha$ pathways, we proposed to further elucidate crocin's pharmacological mechanism. Adult male Sprague-Dawley rats were randomly divided into five groups: control group, hypoxia group (rats were rapidly transported to high altitude of $4200 \mathrm{~m}$ for $72 \mathrm{~h}$ ), and crocins +hypoxia groups (pretreatment with crocin of 25,50 , and $100 \mathrm{mg} / \mathrm{kg} / \mathrm{d}$ for 3 days). The learning and memory ability was tested by Morris water maze analysis. Hippocampal histopathological changes were observed by HE staining and Nissl staining. The expression of NRF1, TFAM, Bcl-2, Bax, and caspase-3 was detected by immunohistochemistry, RT-PCR, and western blotting test. The contents of malondialdehyde (MDA), superoxide dismutase (SOD), glutathione (GSH), and glutathione peroxidase (GSHPx) were detected by the TBA, WST, and colorimetry method. Neuronal apoptosis was observed by TUNEL staining. After crocin pretreatment, the traveled distance was significantly reduced and the percentage of time in the target quadrant was significantly increased tested by Morris water maze. And neuronal damage in the hippocampus was also significantly ameliorated based on HE staining and Nissl staining. Furthermore, in hippocampus tissue, mitochondrial biosynthesis-related factors of NRF1, TFAM expression was increased; oxidative stress factors of SOD, GSH, and GSHPx expression level were increased, and MDA and glutathione disulfide (GSSG) level were decreased; antiapoptotic protein Bcl-2 expression was increased, and proapoptotic proteins Bax and caspase-3 expression were decreased, with a manner of crocin dose dependent. Therefore, the cognitive protective mechanism of crocin in rat under acute hypoxia was related to promoting mitochondrial biosynthesis, ameliorating oxidative stress injury, and decreasing neuronal apoptosis.

\section{Introduction}

In the high-altitude area, the atmospheric pressure drops and the oxygen content is low, which causes a decrease in the partial pressure of oxygen at every point along the oxygen transport cascade from ambient air to cellular mitochondria. Acute high-altitude hypoxia affects the blood flow and its consequent distribution to organs and the efficiency of $\mathrm{O}_{2}$ utilization. And the brain, which is the most susceptible organ to hypoxic damage due to its high energy demands, is very vulnerable to hypoxia which could cause headaches, dizziness, blurred vision, tinnitus, spatial learning and memory impairment, and even pathological changes [1-3]. Additionally, high-altitude hypoxia affects severely the structural integrity of the principal neurons in the hippocampus, resulting in decreased hippocampus-dependent learning and 


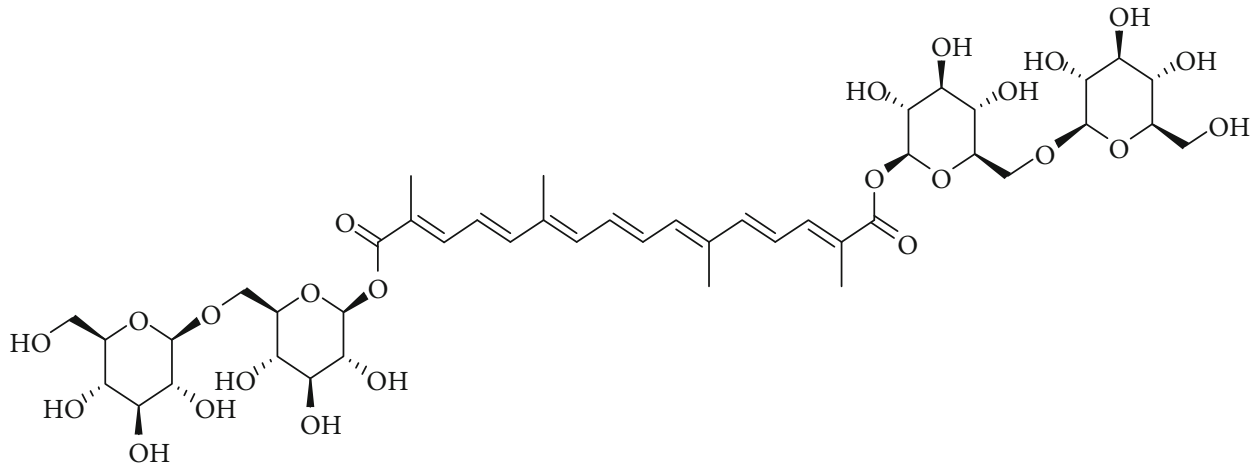

Figure 1: Chemical structure of crocin.

memory function $[4,5]$. The pathological features of hippocampus injury induced by acute hypobaric hypoxia include mitochondrial dysfunction, morphological changes, and upregulation of genes associated with apoptosis [6]. In addition, hypoxia also induces an imbalance between free radical generation and antioxidant protection, resulting in an oxidative damage of biomolecules $[7,8]$. Therefore, it is of utmost importance to provide protective measures against brain damage due to acute hypoxia. The treatment or improvement of central nervous system damage due to acute high-altitude hypoxia has recently attracted increasing attention in the field of high-altitude medicine $[9,10]$. SIRT1 (silent information regulatory factor 1) deacetylation of PGC- $1 \alpha$ (peroxisome proliferator-activated receptor gamma coactivator- $1 \alpha$ ) has been extensively implicated in mitochondrial biogenesis, oxidation stress, and apoptosis $[11,12]$. Studies showed that upregulating the expressions of SIRT1 and PGC- $1 \alpha$ level could preserve mitochondrial function and attenuate oxidative stress injury [13] by increasing NRF1 (nuclear respiratory factor 1) and TFAM (mitochondrial transcription factor A) protein expressions, enhancing SOD activities and suppressing the contents of ROS and MDA [14].

The drugs currently used to prevent acute altitude sickness include acetazolamide, dexamethasone, montelukast, and aspirin, but these drugs have varying degrees of side effects, including sensory abnormalities, gastrointestinal bleeding, osteoporosis, and increased risk of infection [15]. Plants are excellent sources of bioactive compounds throughout history in the search for new drugs. Crocin, a watersoluble carotenoid, is regarded as the quality marker for the quality control of saffron, which has been extensively used for the treatment of brain and cardiovascular diseases in traditional Tibetan medicine (Chinese Pharmacopoeia Commission, 2015, Figure 1). Crocin showed the effects of antiapoptosis, antioxidation, neuroprotection, and inhibition of mitochondrial dysfunction [16-18]. Our previous study found that crocin pretreatment improved learning and memory in rat under acute high-altitude hypoxia, and it could upregulate the level of SIRT1 and PGC- $1 \alpha$ in the hippocampus [19]. But the further cognitive protective mechanism was poorly understood.

In this article, we focused on key factors regulated by the SIRT1/PGC-1 $\alpha$ pathway, mainly concerned with mito- chondrial biosynthesis (NRF1 and TFAM), oxidation stress (SOD, GSH, GSSG, GSHPx, and MDA), and apoptosis (Bax, Bcl-2, and caspase-3), to further illustrate the underling brain protective mechanism of crocin under acute hypoxia exposure.

\section{Materials and Methods}

2.1. Medicine and Reagents. Crocin was purchased from Sigma-Aldrich (purity above 98\%; Sigma-Aldrich, Japan; catalog \#BCBV9507) (Figure 1) and dissolved in normal saline. Hematoxylin-eosin (catalog \#AR1180-100), rabbit antiTFAM (antibody catalog \#PB0413), and BCA Protein Assay Kit (catalog \#AR1189) were purchased from Boster Biological Technology (Wuhan, China). Trizol reagent (catalog \#15596-026) was purchased from Invitrogen; Nissl solution (catalog \#C0117) was purchased from Beyotime Biotechnology (Beijing, China). Rabbit anti-NRF1 (antibody catalog \#ab175932), rabbit anti-Bcl-2 (antibody catalog \#ab194583), rabbit anti-Bax (antibody catalog \#ab53154), rabbit anticaspase-3 (antibody catalog \#ab4051), and rabbit antiGAPDH (antibody catalog \#ab9485) antibodies were supplied from Abcam Biotechnology (Cambridge, MA, USA). Goat anti-rabbit IgG-HRP (catalog \#BA1056) were purchased from Boster Biological Technology (Wuhan, China). SOD (catalog \#A001-3-2), GSH (catalog \#A006-2-1), GSHPx (catalog \#A005-1-2), MDA (catalog \#A003-1-2), and GSSG (catalog \#A061-1-1) commercial kits were obtained from Jiancheng Biological Technology (Nanjing, China). Takara PrimeScript RT reagent kit and Takara TB Green ${ }^{\mathrm{TM}}$ Premix Ex $\mathrm{Taq}^{\mathrm{TM}}$ (Tli RNaseH Plus) kit were obtained from Takara Biotechnology Co., Ltd (RR820A, Dalian, China).

2.2. Animals. Adult male SD rats (210-230 g) of specific pathogen free (SPF) were obtained from the Experimental Animal Center of Xi'an Jiaotong University with a certificate number (license No. SCXK 2017-003). All experimental protocols were approved by the Animal Experimentation Ethics Committee of Qinghai University, and appropriate efforts were applied to minimize distress and pain of the experimental animals.

Animal model experiment was based on our previous research method [19]. Briefly, rats were randomly assigned to five experimental groups ( $n=18$, each group): control 
group (Ctr, saline), acute high-altitude hypoxia group ( $\mathrm{AHH}$, saline), low dose of crocin-pretreated hypoxic group (LHG, $25 \mathrm{mg} / \mathrm{kg} / \mathrm{d}$ ), middle dose of crocin-pretreated hypoxic group (MHG, $50 \mathrm{mg} / \mathrm{kg} / \mathrm{d}$ ), and high dose of crocinpretreated hypoxic group (HHG, $100 \mathrm{mg} / \mathrm{kg} / \mathrm{d}$ ). Crocin (dissolved in $1 \mathrm{~mL}$ saline) was administered 3 days before the acute hypoxia exposure by intramuscular drug once per day. The rats in the control group were treated with a corresponding volume of normal saline. Rats were fed in an environmentally controlled room at $20 \pm 2^{\circ} \mathrm{C}$, with free access to food and water. The rats in the acute high-altitude hypoxic group and the crocin-pretreated hypoxic group were exposed to the acute hypoxic environment at $4200 \mathrm{~m}$ for 3 days (the Gande County of Qinghai Province, China), where the air density was $0.802 \mathrm{~kg} / \mathrm{m}^{3}$, approximately $62 \%$ of the air density at sea level. As oxygen varies in direct proportion with air, the oxygen content in the air was also $62 \%$ of that at sea level [19].

\subsection{Morris Water Maze (MWM) Test and Physiological} Index. Rats were subjected to a daily session of four training trials at intervals of $30 \mathrm{~min}$ for five consecutive days [20] at approximately the same time before the induction of acute high-altitude hypoxia. The maze consisted of a round black metal tank of $160 \mathrm{~cm}$ in diameter with a transparent circular platform placed $2 \mathrm{~cm}$ below the water surface and fixed in one of the four quadrants during the training. The trial was stopped once the rats reached the platform. If the rats were unable to reach the platform within $60 \mathrm{~s}$, they were placed on the platform for $10 \mathrm{~s}$ and then removed from the pool. A video camera linked to a computer directly located above the MWM together with an imaging analysis system (Institute of Materia Medica, Chinese Academy of Medical Sciences, China) was used to record the percentage of time spent in the target quadrant, and the traveled distance of the swim path.

After hypoxia exposure for 3 days, rats were weighed and their body weights were recorded. The same group of rats were anesthetized with urethane $(1.0 \mathrm{~g} / \mathrm{kg})$ and sacrificed after the behavioral MWM test. Blood samples $(0.5 \mathrm{~mL})$ were taken from the abdominal aorta and used for blood cell analysis using blood cell counter (BC-5000 Vet, Mindray company, Guangzhou, China). Red blood cells (RBC), platelets (PLT), and white blood cells (WBC) were measured.

One portion of the hippocampus of 6 rats in each group was fixed in $4 \%$ paraformaldehyde solution, while the other portion was fixed in 3\% glutaraldehyde for further histopathological assessment and immunohistochemical test, respectively. The hippocampus of the remaining rats was flashfrozen in liquid nitrogen and stored at $-80^{\circ} \mathrm{C}$ for western blotting, qRT-PCR, and antioxidation index analysis.

2.4. HÆE Staining. Brain tissues were prefixed in $4 \%$ paraformaldehyde, subsequently washed in running tap water for $24 \mathrm{~h}$ and then subjected to routine dehydration and paraffin embedding. Brain tissues were cut into $3 \mu \mathrm{m}$ thick serial sections by Leica microtome (Leica, Germany). Brain sections were then deparaffinized in xylene, rehydrated via graded alcohols, and stained with $\mathrm{H} \& \mathrm{E}$, according to a standard pro- tocol. Pathological changes were observed under an optical microscope (Leica, Heidelberg, Germany).

2.5. Nissl Staining. Brain sections were dewaxed and then placed in deionized water for $1 \mathrm{~min}$, transferred to a Nissl solution at $37^{\circ} \mathrm{C}$ for $5 \mathrm{~min}$ to achieve an optimal staining, and quickly washed in deionized water. Next, slides were immersed in $70 \%$ ethanol for 5 second twice and mounted on slide using neutral gum; sections were photographed by the Leica microscope. Image-Pro Plus 6.0 software (Media Cybernetics Inc., Maryland, USA) was used to analyze the image and calculate the IOD value. The IOD value of each slice $=$ sum of three discontinuous visual field IOD value/3.

2.6. TBA, WST, and Colorimetry Method. The liquid nitrogen frozen complete hippocampal tissues were placed on ice and homogenized. The homogenates were centrifuged at low temperature at $13000 \mathrm{~g}$ for $15 \mathrm{~min}$. The supernatant was collected and protein quantified, hippocampus SOD was determined by water-soluble tetrazolium-1 (WST-1), MDA was determined by thiobarbituric acid (TBA) colorimetry, GSHPx activity was based on the consumption of reduced glutathione, and GSH/GSSG content was detected by dithio-bis-nitrobenzoic acid (DTNB) (Jiancheng Bioengineering Institute Co., Ltd., Nanjing, China).

2.7. Immunohistochemical Staining. The whole hippocampus was cut at a thickness of approximately $3 \mu \mathrm{m}$. Sections were deparaffinized with xylene and rehydrated with ethanol. Sections were incubated overnight at $4{ }^{\circ} \mathrm{C}$ with the following primary antibodies: rabbit anti-rat, NRF1 (1:50), TFAM (1:50), Bcl-2 (1:200), Bax $(1: 200)$, and caspase-3 $(0.002 \mathrm{mg} / \mathrm{mL})$. The sections were rinsed in PBS 3 times for $5 \mathrm{~min}$, incubated with the appropriate biotinylated secondary antibody followed by an avidin-biotin horseradish peroxidase complex (Boster Biological Technology, Wuhan, China), with diaminobenzidine (DAB) as substrate. All incubations were performed in a humidified chamber. The negative control sections from each animal were subjected to the same staining procedure, except that the primary or secondary antibody was omitted. Sections were photographed by the Leica microscope; three random and nonoverlapping positively stained microscopic fields at 400x magnification were examined in each section of the hippocampal CA1 region. The true color image analysis Image-Pro Plus 6.0 software (Media Cybernetics Inc., Maryland, USA) was applied to determine the integral optical density (IOD) values of protein (IOD value of each slice $=$ sum of three discontinuous visual field IOD value/3).

2.8. Western Blotting. Total proteins were isolated from the frozen hippocampus. The hippocampus was homogenized in Radio Immuno Precipitation Assay (RIPA) buffer (50 mM Tris ( $\mathrm{pH} 8.0$ ), $150 \mathrm{mM}$ sodium chloride, $0.1 \%$ sodium dodecyl sulphate (SDS), $0.5 \%$ sodium deoxycholate, and $1 \%$ Triton X-100) mixed with phenylmethanesulfonyl fluoride (PMSF) and then sonicated for ice cold water. The homogenates were centrifuged at $2000 \mathrm{~g}$ for $20 \mathrm{~min}$ at $4^{\circ} \mathrm{C}$, and protein concentration of the supernatant was quantified using the BCA method. Adjust the protein concentration to 
TABLE 1: List of primers used for qRT-PCR.

\begin{tabular}{|c|c|c|c|}
\hline Gene symbol & GenBank accession & Sequence $\left(5^{\prime}\right.$ to $\left.3^{\prime}\right)$ & Product length $(\mathrm{bp})$ \\
\hline \multirow{2}{*}{ NRF1 } & \multirow{2}{*}{ NC_000007.14 } & F:TTACTCTGCTGTGGCTGATGG & \multirow[t]{2}{*}{143} \\
\hline & & R:CCTCTGATGCTTGCGTCGTCT & \\
\hline \multirow{2}{*}{ TFAM } & \multirow{2}{*}{ NM_031326 } & F:TGAAGCTTGTAAATCAGGCTTGGA & \multirow[t]{2}{*}{146} \\
\hline & & R:GAGATCACTTCGCCCAACTTCAG & \\
\hline \multirow{2}{*}{$B c l-2$} & \multirow{2}{*}{ NM_016993.1 } & F:GACTGAGTACCTGAACCGGCATC & \multirow[t]{2}{*}{135} \\
\hline & & R:CTGAGCAGCGTCTTCAGAGACA & \\
\hline \multirow{2}{*}{ Bax } & \multirow{2}{*}{ NM_017059.2 } & F:TGGCGATGAACTGGACAACAA & \multirow[t]{2}{*}{65} \\
\hline & & R:GGGAGTCTGTATCCACATCAGCA & \\
\hline \multirow{2}{*}{ Caspase-3 } & \multirow{2}{*}{ NM_012922.2 } & F:GCAGCAGCCTCAAATTGTTGAC & \multirow[t]{2}{*}{144} \\
\hline & & R:TGCTCCGGCTCAAACCATC & \\
\hline \multirow{2}{*}{$\beta$-Actin } & \multirow{2}{*}{ NM_031144.2 } & F:CCTAAGGCCAACCGTGAAAA & \multirow[t]{2}{*}{103} \\
\hline & & R:CAGAGGCATACAGGGACAACAC & \\
\hline
\end{tabular}

$2 \mu \mathrm{g} / \mu \mathrm{L}$ for sample preparation, equal amounts of protein $(20 \mu \mathrm{g})$ were loaded in each lane of $10 \%$ SDSpolyacrylamide gels. After the electrophoresis, proteins were transferred to nitrocellulose membranes and incubated with $5 \%$ skim milk for $2 \mathrm{~h}$ at room temperature. Membranes were incubated overnight at $4^{\circ} \mathrm{C}$ with the following primary antibodies: rabbit anti-rat NRF1 $(1: 1000)$, rabbit anti-rat TFAM $(1: 1000)$, rabbit anti-rat Bcl-2 $(1: 2000)$, rabbit anti-rat Bax $(1: 2000)$, rabbit anti-rat caspase-3 $(1: 500)$, and rabbit antirat $\beta$-actin $(1: 2500)$ used as the loading control. The membrane was washed 3 times with PBS for 5 min each time and exposed to a horseradish peroxidase-conjugated goat anti-rabbit secondary antibody $(1: 5000)$ for $1 \mathrm{~h}$ at room temperature. The membrane was then developed using enhanced chemiluminescence (ECL, Santa Cruz Biotechnology Inc.), followed by autoradiography. The films were scanned, and the optical density was determined using the TANON GIS analysis system.

2.9. Quantitative Real-Time PCR ( $q R T-P C R)$. Total RNA was isolated from the frozen hippocampus using TRIZOL reagent. cDNA was obtained using a Takara PrimeScript $\mathrm{RT}$ reagent kit according to the manufacturer's protocol. mRNA of NRF1, TFAM, Bax, Bcl-2, and caspase-3 was determined by the Takara TB Green ${ }^{\mathrm{TM}}$ Premix Ex Taq ${ }^{\mathrm{TM}}$ (Tli RNaseH Plus) kit (RR820A) according to the manufacturer's instructions with the ABI7500Real-Time PCR system (Bio-Rad, CA, USA). The PCR reaction system was $25 \mu \mathrm{L}$ (SYBR Premix Ex Taq II (Tli RNaseH Plus, 2x) $12.5 \mu \mathrm{L}$, Primer $\mathrm{F}(10 \mu \mathrm{M}) 1 \mu \mathrm{L}$, Primer $\mathrm{R}(10 \mu \mathrm{M})$ $1 \mu \mathrm{L}$, cDNA $2 \mu \mathrm{L}$, and $\mathrm{dH}_{2} \mathrm{O} 8.5 \mu \mathrm{L}$ ). The PCR conditions were as follows: $95^{\circ} \mathrm{C}$ for 30 seconds and 40 cycles of $95^{\circ} \mathrm{C}$ for 5 seconds, $60^{\circ} \mathrm{C}$ for 34 seconds, followed by a melting curve analysis $\left(95^{\circ} \mathrm{C}\right.$ for 15 seconds, $60^{\circ} \mathrm{C}$ for 1 minute, and then $95^{\circ} \mathrm{C}$ for 30 seconds and $60^{\circ} \mathrm{C}$ for 15 seconds). The sequences of the forward and reverse primers, purchased from Takara Biotechnology, used in this study are summarized in Table 1. Gene expression was normalized to $\beta$-actin and calculated by the $2-\Delta \Delta \mathrm{ct}$ method. Primers were amplified with approximately equal efficiencies.

2.10. TUNEL Assay. Brain sections were deparaffinized in xylene and rehydrated via graded alcohols. Sections were hydrated and hydrolyzed by proteinase $\mathrm{K}$ working solution for $10 \mathrm{~min}$. After being washed with PBS for 3 times, sections were treated with $20 \%$ normal bovine serum and incubated for $30 \mathrm{~min}$. Then, the sections were treated with TdT buffer for $5 \mathrm{~min}$ and incubated in a humid chamber for $1 \mathrm{~h}$ after adding $50 \mu \mathrm{L}$ TdT reaction solution dropwise. Then, sections were kept warm in prewarmed washing buffer at $37^{\circ} \mathrm{C}$ for 30 min. After being washed with PBS for 3 times, sections were treated with $\mathrm{DAB}$ and incubated at $37^{\circ} \mathrm{C}$ for $5 \mathrm{~min}$. TUNEL-positive cells were stained with a brown-yellow color and were observed under a light microscope $(\times 400$ magnification). Three nonoverlapping fields were randomly selected and photographed to record the total number of TUNEL-positive cells. TUNEL-positive cells (\%) were calculated as follows: (positive staining cells (brown)/total cells) $\times 100 \%$.

2.11. Statistical Analysis. Statistical analysis was performed using SPSS17.0 software. Results were reported as mean \pm SEM. The significance of variables among groups was calculated by one-way ANOVA, followed by the StudentNewman-Keuls test and Dunnett's multiple comparison test. A $p$ value of $<0.05$ was considered statistically significant.

\section{Results}

3.1. Animal Model. We first performed the Morris water maze (MWM) test to evaluate the cognitive performance of different groups of rats (Figure 2). In the acute highaltitude hypoxia group $(\mathrm{AHH})$, the traveled distance in the MWM test was significantly increased; the percentage of time in the target quadrant was significantly decreased $(p<0.05$, vs. control group, Figure 2). The rat model of acute 


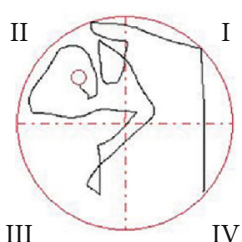

A

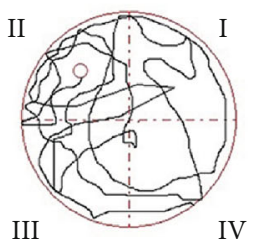

B

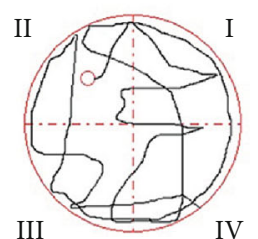

C

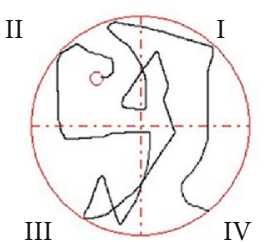

D

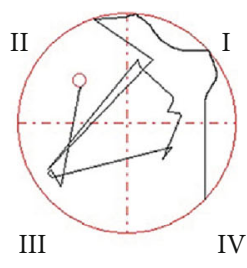

E

(a)

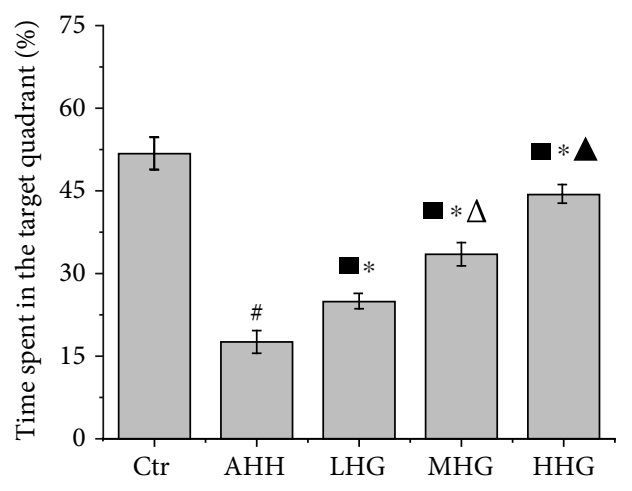

(b)

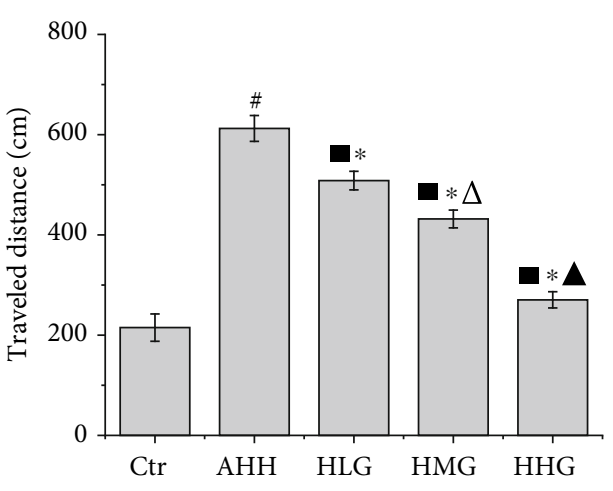

(c)

FIGURE 2: Crocin pretreatment ameliorated learning and memory impairment in acute high-altitude hypoxia rats $(n=12)$. Ctr: control group, with saline. AHH: rats exposed under hypoxia for 3 days at $4200 \mathrm{~m}$. LHG, MHG, HHG: rats exposed under hypoxia for 3 days at $4200 \mathrm{~m}$ after pretreatment by $25 \mathrm{mg} / \mathrm{kg} / \mathrm{d}, 50 \mathrm{mg} / \mathrm{kg} / \mathrm{d}$, and $100 \mathrm{mg} / \mathrm{kg} / \mathrm{d}$ of crocin. (a) Characteristic swimming direction of the rats during the Morris water maze (MWM) test. (A) Ctr; (B) AHH; (C) LHG; (D) MHG; (E) HHG. (b) The percentage of time spent in the target quadrant. (c) Traveled distance. ${ }^{\#} p<0.05$ versus Ctr, ${ }^{*} p<0.05$ versus $\mathrm{AHH},{ }^{\circ} p<0.05$ versus $\mathrm{Ctr},{ }^{\triangle} p<0.05$ versus HLG, and $\boldsymbol{\star}^{\wedge} p 0.05$ versus HMG.

TABLE 2: Effects of crocin pretreatment on physiological indices of rats exposed to acute high hypoxic condition for 3 days (mean \pm SEM, $n=12$ ).

\begin{tabular}{lcccc}
\hline Group & BW $(\mathrm{g})$ & WBC $\left(10^{9} / \mathrm{L}\right)$ & $\mathrm{RBC}\left(10^{12} / \mathrm{L}\right)$ & $\mathrm{PLT}\left(10^{9} / \mathrm{L}\right)$ \\
\hline Ctr & $216.1 \pm 6.2^{\#}$ & $6.7 \pm 0.3^{*}$ & $8.5 \pm 0.5^{\#}$ & $771.5 \pm 58^{\#}$ \\
AHH & $214.8 \pm 5.8$ & $9.0 \pm 0.5$ & $8.8 \pm 0.4$ & $754.3 \pm 47$ \\
HLG & $214.4 \pm 5.2^{\#}$ & $8.1 \pm 0.4$ & $9.1 \pm 0.6^{\#}$ & $773.4 \pm 68$ \\
HMG & $217.4 \pm 4.6^{\#}$ & $6.6 \pm 0.3^{*}$ & $10.1 \pm 0.5^{\#}$ & $723.4 \pm 75^{\#}$ \\
HHG & $218.8 \pm 6.3^{\#}$ & $7.3 \pm 0.4^{*}$ & $9.9 \pm 0.6^{\#}$ & $751.6 \pm 67^{\#}$ \\
\hline
\end{tabular}

Ctr: control group, with saline; AHH: rats exposed under hypoxia for 3 days at $4200 \mathrm{~m}$; LHG, MHG, HHG: rats exposed under hypoxia for 3 days at $4200 \mathrm{~m}$ after pretreatment by $25 \mathrm{mg} / \mathrm{kg} / \mathrm{d}, 50 \mathrm{mg} / \mathrm{kg} / \mathrm{d}$, and $100 \mathrm{mg} / \mathrm{kg} / \mathrm{d}$ of crocin; BW: body weight; RBC: red blood cell; WBC: white blood cell; PLT: platelet. ${ }^{*} p<0.05$ vs. AHH; ${ }^{*} p>0.05$ vs. AHH.

hypoxia-induced cognitive impairment was successfully established.

3.2. Animal Characteristics. There were no significant differences of body weight a in each group ( $p>0.05$, Table 2$)$. The blood routine examination showed a significant increase in WBC in the AHH group when compared with that in the Ctr group $(p<0.05$, Table 2). The MHG and HHG groups showed a significant decrease in WBC and increase in PLT when compared with the AHH group $(p<0.05$, Table 2$)$. However, RBC and PLT had no significant differences each group $(p>0.05$, Table 2$)$.
3.3. Crocin Pretreatment Improved Spatial Memory in Acute High-Altitude Hypoxia Rats. We found a significant increased percentage of time spent in the target quadrant and decreased traveled distance in the group pretreated with crocin compared with the AHH group ( $p<0.05$, Figures $2(a)-2(c))$. Our results suggested that crocin pretreatment improved learning and memory performance in acute high-altitude hypoxia rats in a dose-dependent manner.

3.4. Effect of Crocin Pretreatment on Histopathological Changes of the Hippocampus in Acute High-Altitude Hypoxia Rats. The hippocampal neurons of the control group showed an ordered arrangement, normal structure, clear nuclei, and distinct nucleoli. The hippocampal neurons in the CA1 region showed a distinct and regular structure and were densely and clearly arranged in the control group (Figure 3(a)). However, the structure of the hippocampal neurons in the CA1 region in the AHH group was severely damaged compared with the control group. In the $\mathrm{AHH}$ group, the number of cells was reduced, the arrangement of the cells was chaotic, and the neurons were shrunk, nuclear pyknosis, and with an eosinophilic cytoplasm. Crocin pretreatment at all the doses alleviated the pathological damage of hippocampal neurons.

Neurons appeared in a dense arrangement, and the Nissl bodies were abundant in the CA1 region of the hippocampus of the control group (Figures 3(b) and 3(c)). The AHH group showed a reduced amount of Nissl bodies $(71.01 \pm 4.85)$ compared with the control group (340.29 \pm 9.02$)$, with a significantly decreased IOD value $(p<0.05)$. However, the IOD 

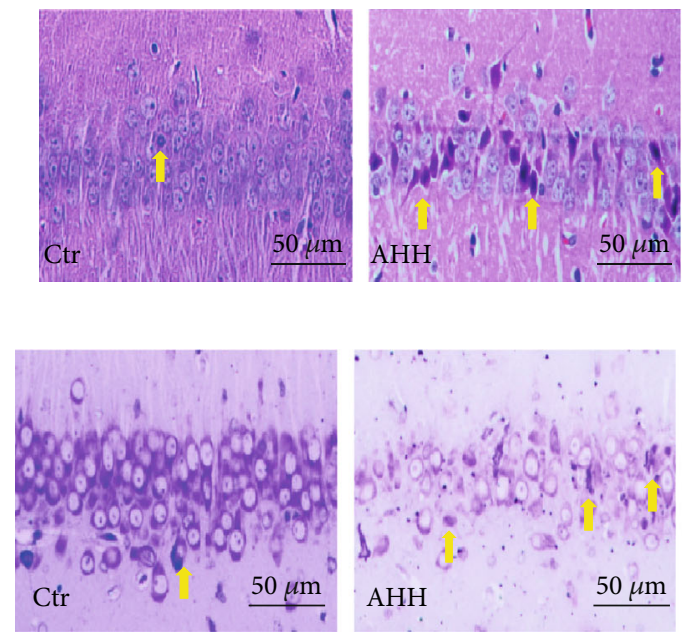
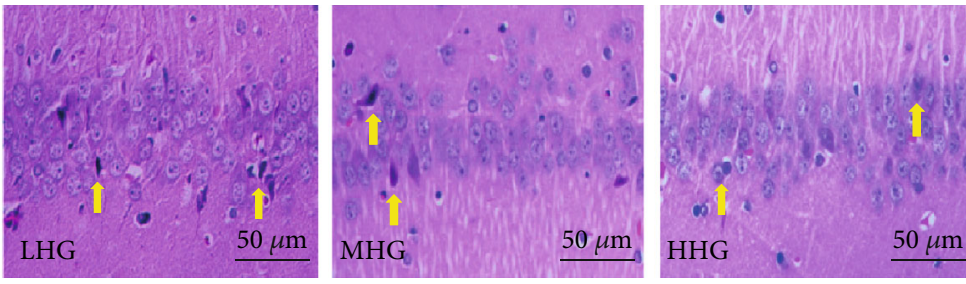

(a)
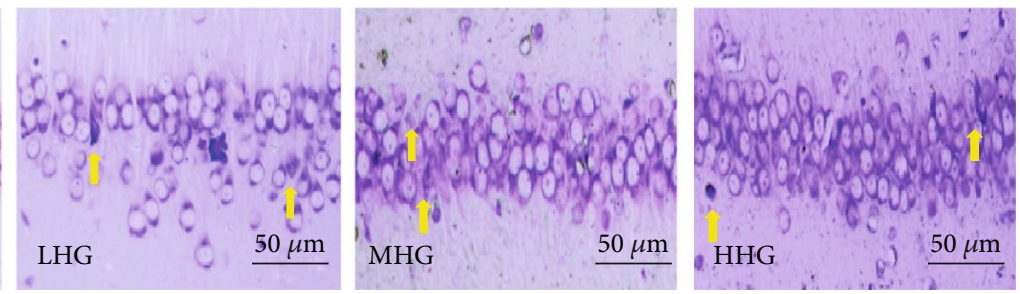

(b)

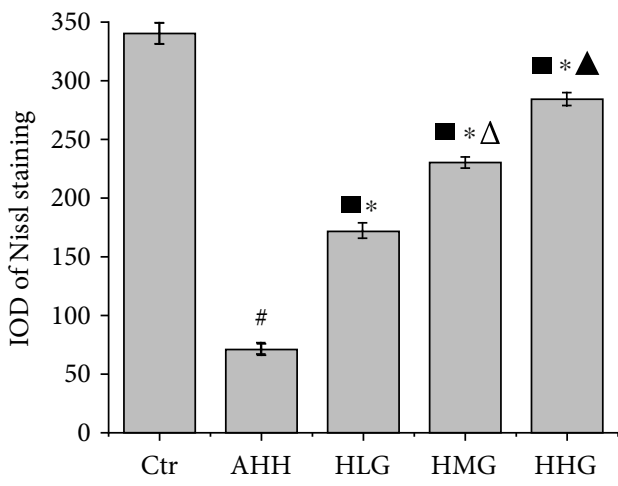

(c)

FIGURE 3: Crocin pretreatment improved the neuronal morphology of the hippocampus in rats under acute high-altitude hypoxia $(n=6$, $\times 400$ magnification, scale bar: $50 \mu \mathrm{m}$ ). Ctr: control group, with saline. AHH: rats exposed under hypoxia for 3 days at $4200 \mathrm{~m}$. LHG, MHG, HHG: rats exposed under hypoxia for 3 days at $4200 \mathrm{~m}$ after pretreatment by $25 \mathrm{mg} / \mathrm{kg} / \mathrm{d}, 50 \mathrm{mg} / \mathrm{kg} / \mathrm{d}$, and $100 \mathrm{mg} / \mathrm{kg} / \mathrm{d}$ of crocin. (a) HE-stained sections across the hippocampal CA1 region. (b, c) Nissl-stained sections across the hippocampal CA1 region. ( $\uparrow$ neurons

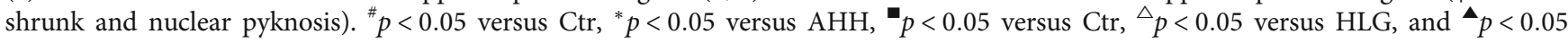
versus HMG.

value was significantly increased in the crocin pretreatment groups at all the used concentrations compared with the AHH group $(p<0.05)$. Pretreatment of crocin significantly inhibited neuronal loss and prevented neuronal Nissl bodies' reduction in the hippocampus. The IOD value in the HHG group $(284.23 \pm 5.67)$ was significantly higher than that in the HLG $(171.65 \pm 6.59)$ and HMG $(230.37 \pm 4.76)$ groups $(p<0.05)$. Crocin pretreatment significantly ameliorated the structural damage of hippocampal cells in rat under acute high-altitude hypoxia exposure.

\subsection{Crocin Pretreatment Improves the Expression of} Mitochondrial Biosynthesis-Related Factors in Rat Hippocampal Cells under Acute High-Altitude Hypoxia. Our previous studies show that in the AHH group, obvious injuries occurred, including a remarkable cell loss and karyopyknosis, swollen and decreased mitochondria, without cristae and with a fragmented double membrane structure. Crocin pretreatment significantly reduced injuries compared with the $\mathrm{AHH}$ group. In all crocin-treated groups, the number of mitochondria was significantly increased. The mitochondrial injury, including swelling, crest disappearance, and membrane structure damage, was ameliorated, suggesting a protection of the mitochondrial structure [19]. In this article, the results of real-time PCR and western blot showed that the level of NRF1 and TFAM in the AHH group was significantly decreased than that in the control group in rat's hippocampus $(p<0.05$, Figures $4(\mathrm{a})-4(\mathrm{c}))$. In all crocin-pretreated groups, the level of NRF1 and TFAM was significantly increased than that of the AHH group $(p<0.05)$ in a dose-dependent manner. Meanwhile, we detected the expression of NRF1 and TFAM in the CA1 region of rat's hippocampus by the immunohistochemical test. The results showed that TFAM protein was expressed in cytoplasm and NRF1 protein was expressed in nucleus, showing a dark brown color in positive staining areas (Figures 5(a) and 5(b)). The IOD of NRF1 and TFAM in the AHH group was clearly decreased compared with the control group $(p<0.05)$. After treatment by crocin, the IOD of NRF1 and TFAM was also significantly increased compared with the $\mathrm{AHH}$ group $(p<0.05)$ in a dose-dependent manner. 

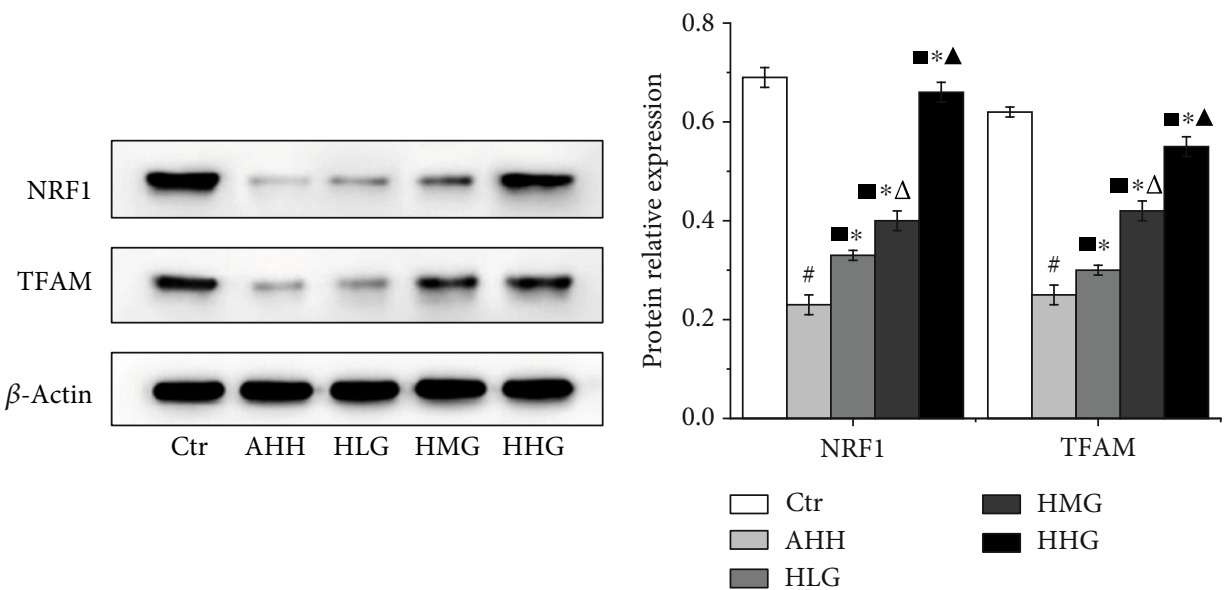

(a)

(b)

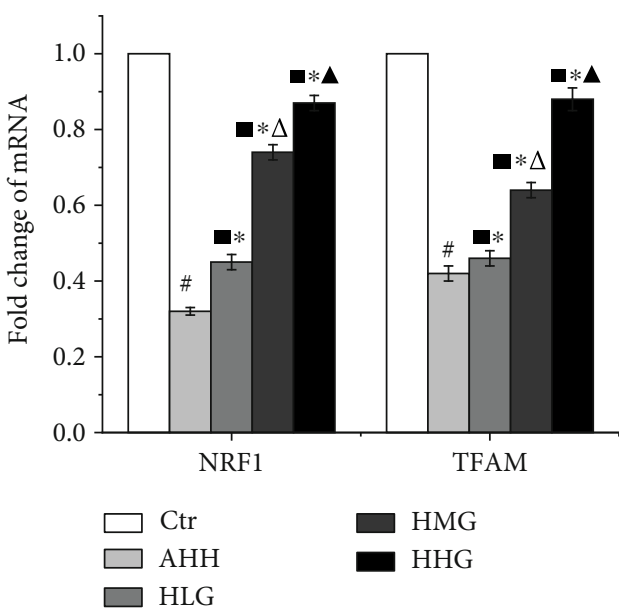

(c)

FIGURE 4: Crocin pretreatment improves the expression of mitochondrial biosynthesis-related factors in rat's hippocampal cells under acute high-altitude hypoxia. Ctr: control group, with saline. AHH: rats exposed under hypoxia for 3 days at $4200 \mathrm{~m}$. LHG, MHG, HHG: rats exposed under hypoxia for 3 days at $4200 \mathrm{~m}$ after pretreatment by $25 \mathrm{mg} / \mathrm{kg} / \mathrm{d}, 50 \mathrm{mg} / \mathrm{kg} / \mathrm{d}$, and $100 \mathrm{mg} / \mathrm{kg} / \mathrm{d}$ of crocin). (a, b) Western blot detection of NRF1 and TFAM protein expression in the hippocampus $(n=12)$. (c) NRF1 and TFAM mRNA expression by qRT-PCR in the hippocampus $(n=12) .{ }^{\#} p<0.05$ versus Ctr, ${ }^{*} p<0.05$ versus AHH, ${ }^{\circ} p<0.05$ versus $\mathrm{Ctr},{ }^{\triangle} p<0.05$ versus HLG, and ${ }^{\boldsymbol{\Delta}} p<0.05$ versus HMG.

\subsection{Crocin Pretreatment Improved the Antioxidant Capacity} in the Hippocampus in Acute High-Altitude Hypoxia Rats. SOD (Figure 6(a)), GSHPx (Figure 6(b)), and GSH (Figure 6(d)) levels were significantly decreased; MDA (Figure 6(c)) and GSSG (Figure 6(e)) concentration was significantly increased in the AHH group; the GSH/GSSG ratio was also significantly reduced ( $p<0.05$, vs. control group). Pretreatment with crocin at all the concentrations significantly reduced the MDA and GSSG level and increased the level of SOD, GSHPx, and GSH, increasing the GSH/GSSG ratio at the same time compared with the $\mathrm{AHH}$ group $(p<0.05)$. So, crocin pretreatment significantly improved the antioxidant capacity of hippocampus in acute highaltitude hypoxia rats.

3.7. Crocin Pretreatment Protected Hippocampal Neurons by Inhibiting Neuronal Apoptosis in Acute High-Altitude Hypoxia Rats. We examined subsequently the expression of $\mathrm{Bcl}-2$, Bax, and caspase- 3 at mRNA and protein levels
(Figures 7(a)-7(c)), and Bcl-2 (Figures $7(\mathrm{a})-7(\mathrm{c})$ ) protein expression in the hippocampus of rats in the $\mathrm{AHH}$ group was significantly lower than that in the control group $(p<0.05)$, while Bax and caspase- 3 were significantly higher than that in the control group $(p<0.05$, Figures $7(\mathrm{a})-7(\mathrm{c}))$. Pretreatment with crocin at all the concentrations significantly increased the Bcl-2 proteins compared with the $\mathrm{AHH}$ group, while crocin decreased the level of Bax and caspase- 3 protein expression $(p<0.05)$ in a dose-dependent manner. Meanwhile, we detected the expression of Bcl-2, Bax, and caspase- 3 in hippocampal neurons in the CA1 area by the immunohistochemical test. Bcl-2, Bax, and caspase- 3 (Figures 8(a) and 8(b)) proteins were expressed in the cytoplasm, showing a dark brown color in positive staining areas. The IOD of Bcl-2 was decreased, and the IOD of Bax and caspase- 3 was increased significantly $(p<0.05$, AHH group vs. control group). The IOD of Bcl-2 was also significantly increased in the crocin-pretreated groups $(p<0.05)$ compared with that in the AHH group, while IOD of Bax and 

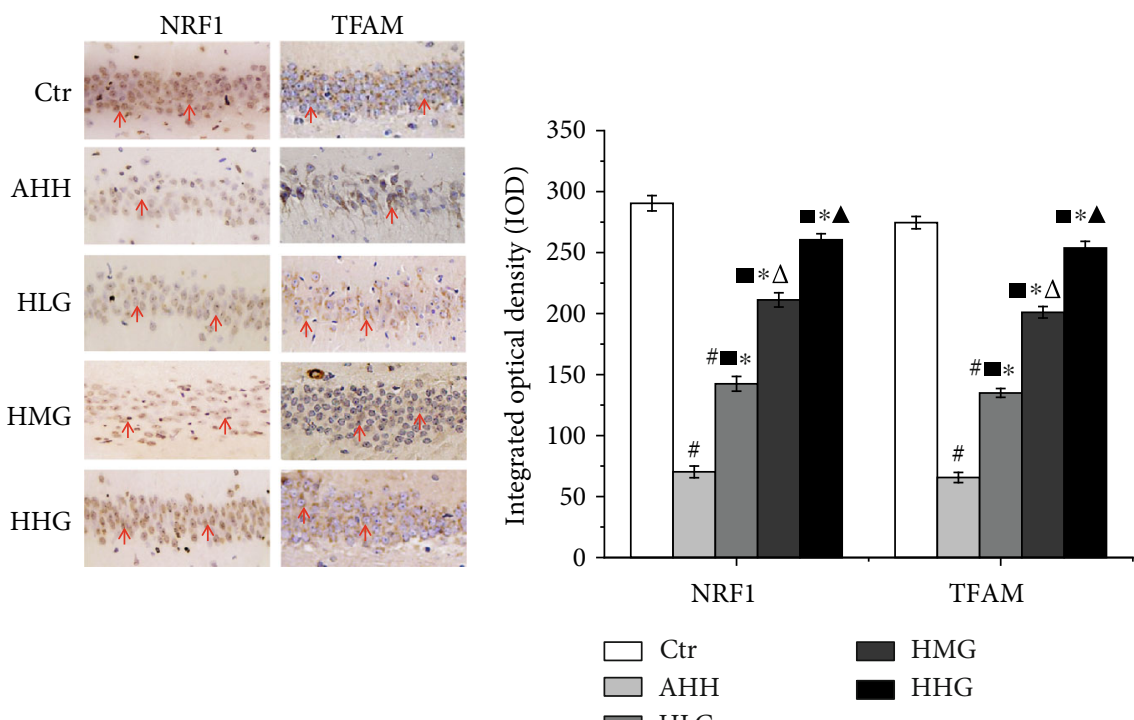

(a)

(b)

FIGURE 5: Crocin pretreatment improves the expression of NRF1 and TFAM in rat's hippocampal cells under acute high-altitude hypoxia. Ctr: control group, with saline. AHH: rats exposed under hypoxia for 3 days at $4200 \mathrm{~m}$. LHG, MHG, HHG: rats exposed under hypoxia for 3 days at $4200 \mathrm{~m}$ after pretreatment by $25 \mathrm{mg} / \mathrm{kg} / \mathrm{d}, 50 \mathrm{mg} / \mathrm{kg} / \mathrm{d}$, and $100 \mathrm{mg} / \mathrm{kg} / \mathrm{d}$ of crocin. (a, b) Immunohistochemical staining of NRF1 and TFAM expression in the CA1 region of the hippocampus $(n=6) .{ }^{*} p<0.05$ versus Ctr, ${ }^{*} p<0.05$ versus AHH, ${ }^{*} p<0.05$ versus Ctr, ${ }^{\triangle} p<0.05$ versus HLG, and ${ }^{\star} p<0.05$ versus HMG.

caspase- 3 was significantly decreased $(p<0.05)$ in a dosedependent manner.

As shown in the results of TUNEL staining, the number of TUNEL-positive cells in the hippocampal CA1 region of the AHH group was significantly increased compared with that of the control group ( $p<0.05$, Figure 9(a)). Pretreatment with crocin significantly reduced the number of positive cell (Figure 9(b)). Crocin upregulated the expression of $\mathrm{Bcl}-2$, while downregulated the expression of caspase- 3 and Bax in a dose-dependent manner, so as to inhibit the harmful effect of hypoxia on these proteins, suggesting that crocin protected hippocampal neurons from acute hypoxia injury by inhibiting neuron apoptosis.

\section{Discussion}

In our previous studies, we observed that crocin pretreatment improved cognitive function in rat under acute high-altitude hypoxia. However, the mechanism of crocin's brain protection remains unclear.

Crocin is the major component of saffron, with antioxidant and anti-inflammatory properties, and antiapoptotic, anticancer, hypolipidemic, neuroprotective, antiaging, and other broad pharmacological effects [21]. At high altitude, there is a decrease in the barometric pressure and a consequent reduction in the oxygen partial pressure $\left(\mathrm{PO}_{2}\right)$, representing an extreme environmental condition [22]. Hypoxia at high altitude causes an imbalance of oxygenous availability to the tissues, causing severe physiological and psychological dysfunction in humans and other animals. Acute hypoxia severely impairs cognition and learning in humans [23]. Crocin mitigates malathion-induced neurological alterations and cognitive impairment by reducing oxidative stress and inflammatory reaction [24]. In addition, crocin improves cognitive performance and reduces hyperglycemia and oxidative stress in diabetic rats [25]. Our study found that crocin pretreatment could significantly improve learning and memory ability in rats induced by acute high-altitude hypoxia. The MWM test showed a significant decrease in traveled distance and increase percentage of time spent in the target quadrant in the crocin groups compared with the hypoxia group in a dose-dependent manner. Based on these observations, further experiments were conducted in order to clarify the protective mechanism of crocin pretreatment. The cells of the immune system are particularly sensitive to changes in oxidant stress because polyunsaturated fatty acids in their plasma membranes are highly susceptible to oxidative stress [26]. It was found that under the condition of 7576 and $5486.4 \mathrm{~m}$ hypobaric hypoxia, the circulating white blood cells (WBC) of rats increased significantly; therefore, it is speculated that hypoxia will cause oxidative stress, which will increase the number and activity of white blood cells [27]. Moreover, we also found that the number of white blood cells in hypoxic rats increased significantly, and crocin pretreatment could significantly decrease the number of white blood cells.

The hippocampus is an essential area in the brain for learning and memory, so the structure and function of hippocampal neurons are of great concern [28]. It was reported that the hippocampus was seriously damaged, and the number of pyramidal cells was decreased in rat under acute highaltitude hypoxia [29]. Moreover, exposure to hypoxia leads to chromatinic condensation and neurodegeneration in the hippocampus [30]. Our results showed that the neurons in the hippocampus of rats were disordered and deformed, and Nissl body was decreased significantly after $72 \mathrm{~h}$ rapidly of acute hypoxia exposure at high altitude. 


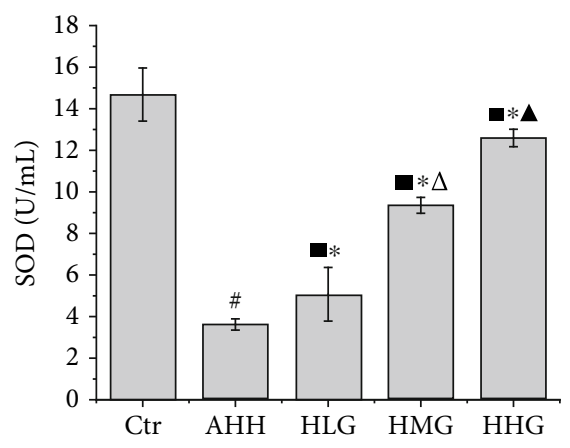

(a)

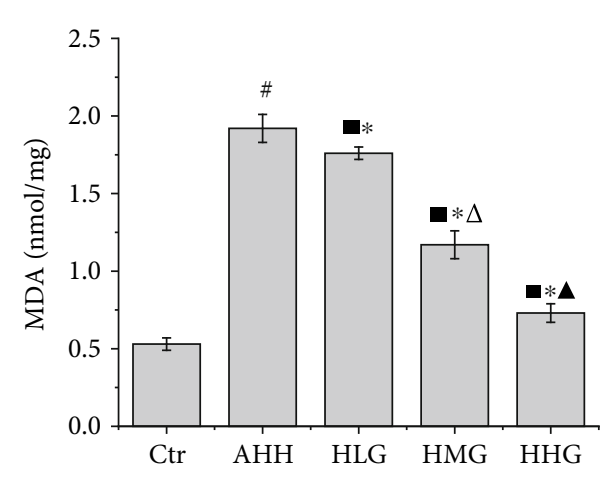

(c)

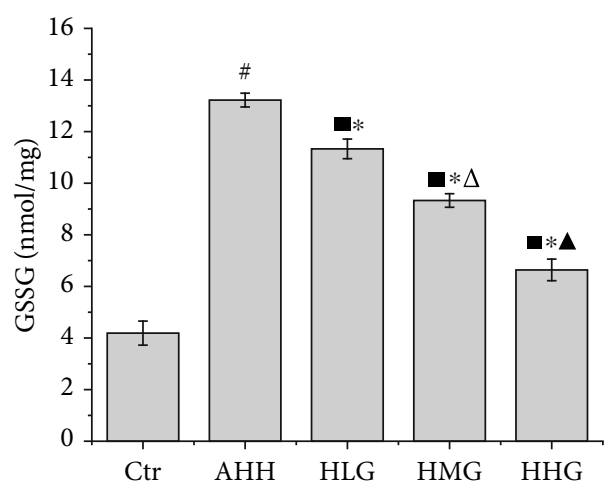

(e)

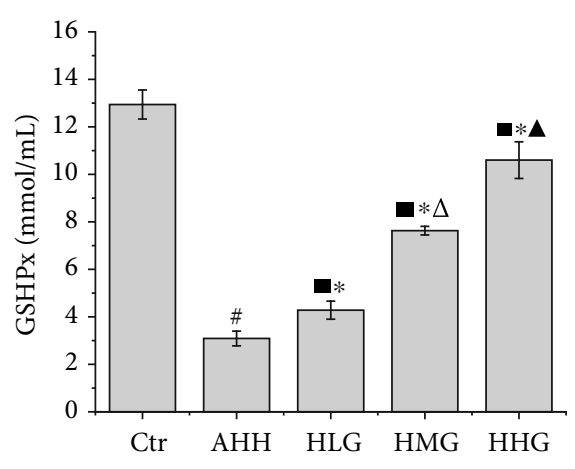

(b)

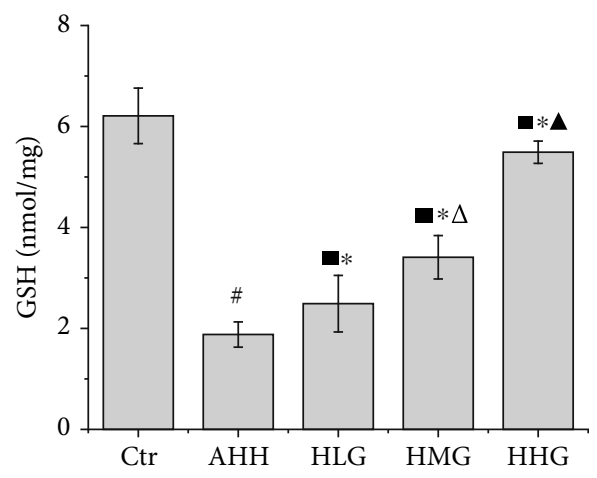

(d)

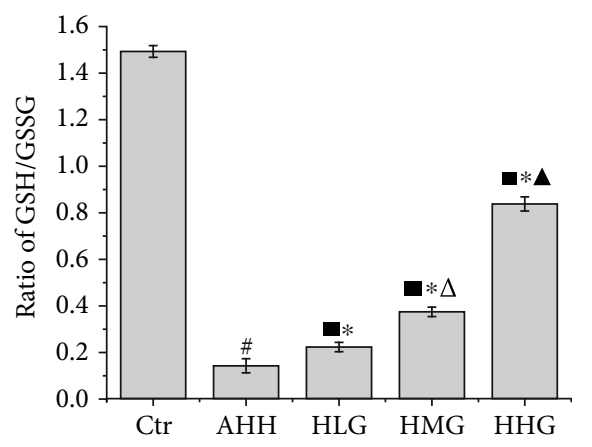

(f)

FIGURE 6: Crocin pretreatment improved the antioxidant capacity in the hippocampus of rats under acute high-altitude hypoxia $(n=12)$. Ctr: control group, with saline. AHH: rats exposed under hypoxia for 3 days at $4200 \mathrm{~m}$. LHG, MHG, HHG: rats exposed under hypoxia for 3 days at $4200 \mathrm{~m}$ after pretreatment by $25 \mathrm{mg} / \mathrm{kg} / \mathrm{d}, 50 \mathrm{mg} / \mathrm{kg} / \mathrm{d}$, and $100 \mathrm{mg} / \mathrm{kg} / \mathrm{d}$ of crocin). (a) The levels of SOD. (b) The levels of GSHPx. (c) The levels of MDA. (d) The levels of GSH. (e) The levels of GSSG. (f) The GSH/GSSG ratio. ${ }^{\#} p<0.05$ versus Ctr, ${ }^{*} p<0.05$ versus AHH, $p<0.05$ versus $\mathrm{Ctr},{ }^{\triangle} p<0.05$ versus $H L G$, and ${ }^{\wedge} p<0.05$ versus $H M G$.

Mitochondrial structural damage and dysfunction are the prominent pathological features in the brain under hypoxia [6]. In our previous study, the ultrastructural observation showed the presence of mitochondrial swelling and crista disappearance and a reduced number of mitochondria in the hippocampal neurons under acute hypoxia at high altitude; hippocampal neurons of crocin-pretreated groups were significantly protected from these injuries including cell loss and karyopyknosis [19].

SIRT1 plays a critical role in the regulation of various metabolic and pathophysiological processes, such as glycometabolism and lipid metabolism, inflammation, senescence, apoptosis, DNA damage repair, autophagy, oxidative stress, and cancer [31, 32]. Mitochondria enhance neuronal functionality and strengthen their resistance to stress, injury, and disease [33]. Research has discovered that long-term memory formation is mediated by SIRT, and enhancing the SIRT1 expression could resist the neurodegeneration in Huntington's disease (HD) mouse brain [34]. Lack of SIRT1 could damage cognitive function including imaginative memory, space learning, and recognition memory. In contrast, overexpression of SIRT1 could perform normal synaptic plasticity and memory capacity [35]. Moreover, SIRT1 is indispensable for synapse growth as well as memory formation and plasticity. Furthermore, SIRT1 could deacetylate PGC- $1 \alpha$ to activate the PGC- $1 \alpha$-NRF-1-TFAM pathway. 

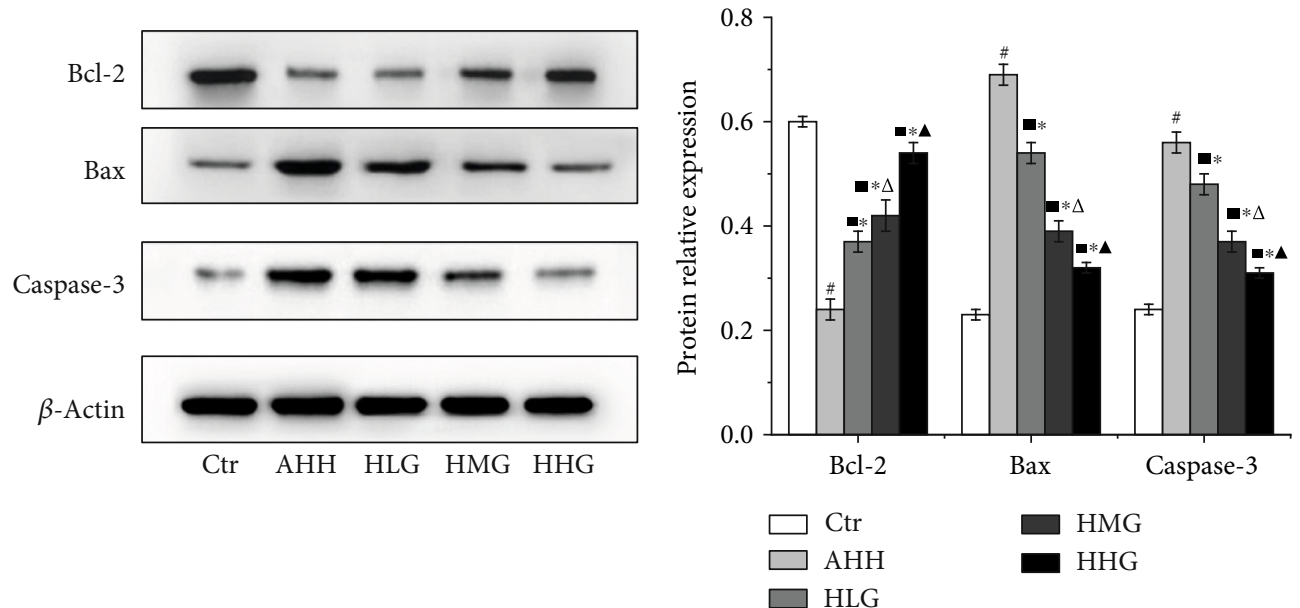

(a)

(b)

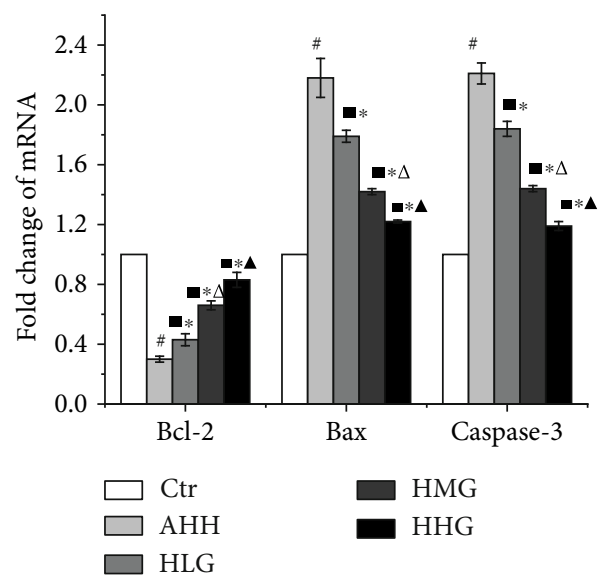

(c)

Figure 7: Crocin pretreatment decreased the Bcl-2, Bax, and caspase-3 expression in the hippocampus of rats under acute high-altitude hypoxia $(n=12)$. Ctr: control group, with saline. AHH: rats exposed under hypoxia for 3 days at 4200 m. LHG, MHG, HHG: rats exposed under hypoxia for 3 days at $4200 \mathrm{~m}$ after pretreatment by $25 \mathrm{mg} / \mathrm{kg} / \mathrm{d}, 50 \mathrm{mg} / \mathrm{kg} / \mathrm{d}$, and $100 \mathrm{mg} / \mathrm{kg} / \mathrm{d}$ of crocin. (a, b) Western blotting detection of Bcl-2, Bax, and caspase-3 protein expression in the hippocampus. (c) Bcl-2, Bax, and caspase-3 mRNA expression by qRTPCR in the hippocampus. ${ }^{\#} p<0.05$ versus $\mathrm{Ctr},{ }^{*} p<0.05$ versus $\mathrm{AHH},{ }^{\boldsymbol{}} p<0.05$ versus $\mathrm{Ctr},{ }^{{ }} p<0.05$ versus HLG, and ${ }^{\star} p<0.05$ versus HMG.

Overexpression of PGC- $1 \alpha$ promotes synaptic differentiation, whereas its downregulation has the opposite effect causing neuropathology [35]. The study found that upregulation of the PGC- $1 \alpha$-NRF1-TFAM pathway promoted mitochondrial biogenesis, alleviated hippocampal neuronal injury, and improved spatial memory deficits in rats with hypoxiaischemia [36]. Mitochondrial biogenesis and function require the coordinated transcription of nuclear and mitochondrial encoded genes and are mediated via transcriptional regulators that respond to extracellular and mitochondrial cues [37]. Therefore, promoting mitochondrial biogenesis plays an important role for neuronal function. Mitochondria are the major ROS producer and exert a crucial role within the cell-mediating processes including apoptosis, detoxification, and $\mathrm{Ca}^{2+}$ buffering [38]. This pivotal role makes mitochondria a potential target to treat a great variety of diseases.

Mitochondrial biogenesis is crucially important in modern neurochemistry because of the broad spectrum of human diseases arising from defects in mitochondrion and ROS homeostasis, energy production, and morphology. Meanwhile, the research found that the biogenesis of mitochondria could be pharmacologically controlled [39]. Several reports indicated that the SIRT1 and PGC- $1 \alpha$ serve as master regulators of mitochondrial biogenesis and function by controlling gene expression [40], while SIRT1, PGC- $1 \alpha$, NRF1, and TFAM promoted the energy synthesis and survival rate of neurons [41]. SIRT1 affects mitochondrial biogenesis by regulating the transcriptional expression of PGC- $1 \alpha$, NRF1, and TFAM [42]. The SIRT1/PGC- $1 \alpha /$ NRF1/TFAM is a central regulator of mitochondrial biosynthesis, and mitochondrial DNA is activated and upregulated by the NRF1 and TFAM [43]. NRF1 is a transcription factor that modulates genes controlling mitochondrial biogenesis and genes involved in diverse cellular functions [44]. According to a published report, upregulating the expression of the SIRT1/PGC$1 \alpha / \mathrm{NRF} 1 / \mathrm{TFAM}$ pathway could increase the expression of mtDNA and mitochondrial biogenesis. In this way, impaired 


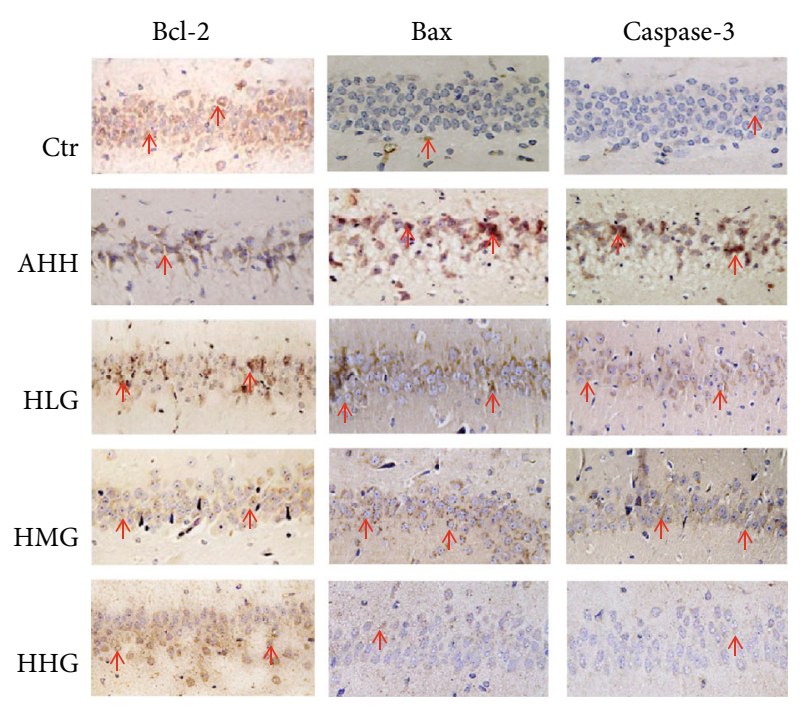

(a)

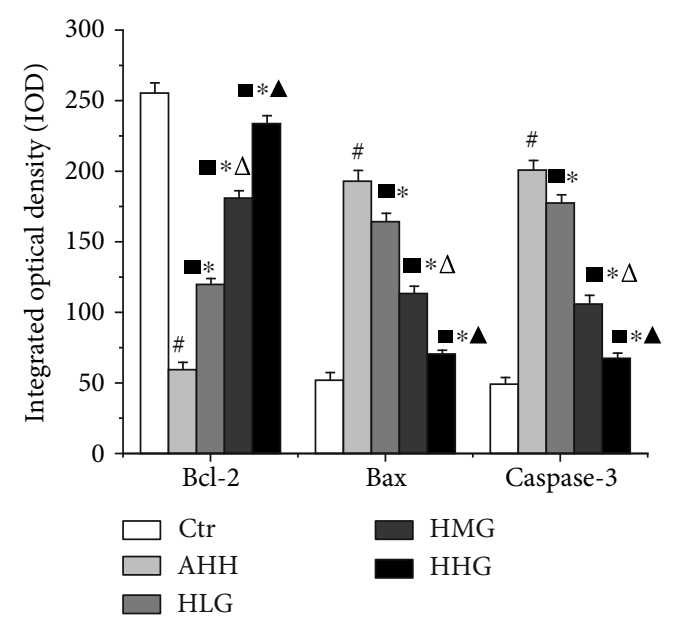

(b)

Figure 8: Crocin pretreatment decreased the Bcl-2, Bax, and caspase-3 expression in the CA1 region of hippocampal neurons under acute high-altitude hypoxia $(n=6)$. Ctr: control group, with saline. AHH: rats exposed under hypoxia for 3 days at $4200 \mathrm{~m}$. LHG, MHG, HHG: rats exposed under hypoxia for 3 days at $4200 \mathrm{~m}$ after pretreatment by $25 \mathrm{mg} / \mathrm{kg} / \mathrm{d}, 50 \mathrm{mg} / \mathrm{kg} / \mathrm{d}$, and $100 \mathrm{mg} / \mathrm{kg} / \mathrm{d}$ of crocin. (a, b) Proteins were expressed in the cytoplasm, showing a dark brown color in positive staining areas. ${ }^{\#} p<0.05$ versus $\mathrm{Ctr},{ }^{*} p<0.05$ versus $\mathrm{AHH}$, - $p<0.05$ versus Ctr, ${ }^{\triangle} p<0.05$ versus HLG, and ${ }^{\boldsymbol{\Delta}} p<0.05$ versus HMG.
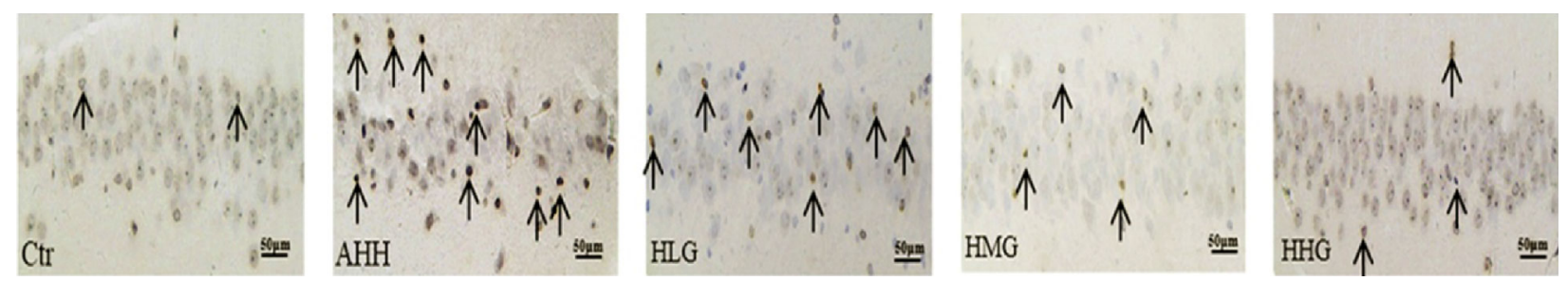

(a)

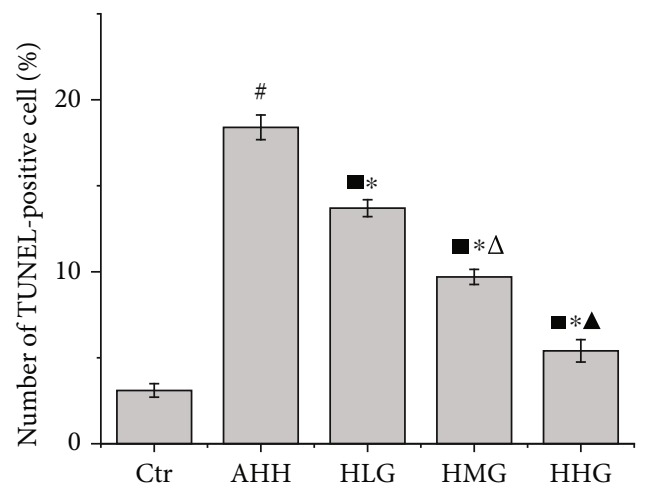

(b)

FIGURE 9: Crocin pretreatment inhibited neuronal apoptosis in the CA1 region of hippocampal neurons under acute high-altitude hypoxia $(n=6)$. Ctr: control group, with saline. AHH: rats exposed under hypoxia for 3 days at $4200 \mathrm{~m}$. LHG, MHG, HHG: rats exposed under hypoxia for 3 days at $4200 \mathrm{~m}$ after pretreatment by $25 \mathrm{mg} / \mathrm{kg} / \mathrm{d}, 50 \mathrm{mg} / \mathrm{kg} / \mathrm{d}$, and $100 \mathrm{mg} / \mathrm{kg} / \mathrm{d}$ of crocin). (a, b) Quantification of TUNELpositive cells in the hippocampus CA1 region. ${ }^{\#} p<0.05$ versus Ctr, ${ }^{*} p<0.05$ versus AHH, ${ }^{\circ} p<0.05$ versus Ctr, ${ }^{\triangle} p<0.05$ versus HLG, and $\boldsymbol{\Delta} p 0.05$ versus HMG. 
mitochondria might be rescued by the activation of mitochondrial biogenesis or regeneration of new mitochondria [45]. The expression of mitochondrial biogenesis genes is known to be under the control of the PGC- $1 \alpha$ and their activator SIRT1. The increase of PGC- $1 \alpha$ activity could enhance the expression of NRF1 gene, while NRF1 could promote the expression of TFAM which directly stimulates mitochondrial DNA replication and transcription [46]. In our study, NRF1 and TFAM gene and protein expression in the hippocampus of rats were significantly decreased under acute high-altitude hypoxia. Pretreatment with crocin significantly increased the level of SIRT1, PGC- $1 \alpha$, NRF1, and TFAM and reduced the mitochondrial damage significantly, suggesting that crocin's protective effect was through increasing mitochondrial synthesis.

PGC- $1 \alpha$ is one of regulators of mitochondrial biogenesis and function, and it plays a remarkable role in the resistance to oxidative stress and in the maintain of mitochondrial integrity [47]. Nuclear respiratory factor 1 (NRF1), perceived as a protein regulating genes controlling mitochondrial biogenesis, is now widely recognized as a multifunctional protein and as a key player in the transcriptional modulation of genes implicated in various cellular functions, including proliferation, and apoptosis [48]. It was reported that upregulating SIRT1, PGC- $1 \alpha$, NRF1, and TFAM reduced neuronal death by alleviating oxidative stress [49]. In addition, when neurons undergo oxidative stress challenge, increased PGC- $1 \alpha$ regulated the cell response through upregulating the expression of antioxidative enzyme (including SOD) [50]. Increased oxidative stress represents an imbalance between intracellular production of free radicals and the cellular defense mechanisms. The sources of ROS are both extracellular (environment, drugs, or radiation) and intracellular (mitochondria or endoplasmic reticulum) [51]. Mitochondrial damage leading to high levels of ROS or MDA is considered to be one of the indicators of oxidative stress. On the other hand, enzymatic ROS scavenging mechanisms include SOD and glutathione peroxidase acts as the first line of defense against excess ROS [52]. Under hypobaric hypoxia, the antioxidant defense system such as GSH, GSHPx, SOD, and GSH/GSSG levels was significantly decreased in the hippocampus [53]. Crocin has been shown to be an antioxidant and neural protective agent. Crocin treatment has shown to counteract oxidative stress by reducing lipid peroxidation and improving the activity of antioxidant enzymes like SOD in some neurodegenerative disorder $[54,55]$. These antioxidant effects of crocin were more effective than those of a-tocopherol at the same concentration on neuronally differentiated pheochromocytoma (PC-12) cells deprived of serum/glucose [56]. In a rat model of malathion-induced depressive-like behavior, crocin significantly decreased MDA levels and increased GSH levels in the hippocampus of rats compared with vitamin E [57]. Our study found that under the condition of acute hypoxia at high altitude, the hippocampus was in a state of high oxidative stress. The pretreatment of crocin played a good role in antioxidant stress; the protective effect of crocin was more obvious at higher doses especially at $100 \mathrm{mg} / \mathrm{kg} / \mathrm{d}$.
According to the published result, the activation of SIRT1 promotes the recovery of mitochondrial protein and function by increasing the biosynthesis of mitochondria and reduces neuronal apoptosis through the PGC- $1 \alpha$ mitochondrial pathway [58]. PGC- $1 \alpha$, NRF1, and TFAM play an antiapoptotic role by decreasing Bax expression and increasing $\mathrm{Bcl}-2$ expression through mitochondrial biosynthesis and antioxidant stress [59]. Exposure to hypoxia increased the number of TUNEL-positive cells along with the increase of caspase- 3 expression in the CA1 region of the hippocampus [60]. Release of cytochrome $c$ into the cytosol during hypoxic stress triggers apoptosis in neuronal cells [61]. Hypoxia increases the levels of caspase- 3 and induces apoptosis in the hippocampal CA1 pyramidal neurons [62]. Moreover, SIRT1 significantly exerted an antiapoptotic effect by deacetylating lysine residue binding to protein kinase $\mathrm{B}$ and decreasing the activity of caspase-3, caspase-9, and related pathways [63]. In our study, crocin played a protective role in hippocampal neuron from oxidative stress damage under acute highaltitude hypoxia in a dose-dependent manner, ensuring neuronic viability and improving GSHPx, GSH, and SOD activity while reducing expression levels of MDA, GSSG, and ratio of GSH/GSSG. Crocin kept high expression of SIRT1, PGC-1 $\alpha$, NRF1, and Bcl-2 and kept a low expression of caspase- 3 and Bax, suggesting that crocin protected hippocampal neurons against acute high-altitude hypoxia injury by inhibiting neuronal apoptosis and activating the SIRT1/PGC-1 $\alpha /$ NRF1/TFAM signaling pathway.

\section{Conclusion}

Our results revealed that crocin significantly reduced hippocampal neuronic damage; improved the histomorphology of hippocampal neurons; markedly kept a high level of GSH, GSHPx, and SOD; reduced MDA and GSSG content; preserved a high SIRT1, PGC- $1 \alpha$, NRF1, TFAM, and Bcl-2 expression; decreased the level of Bax and caspase-3; and reduced the apoptotic rate. Taken together, our study found that crocin could attenuate the damage of hippocampal neurons induced by acute hypoxia by improving mitochondrial structure, reducing oxidative stress injury, and inhibiting neuronic apoptosis via the SIRT1/PGC$1 \alpha /$ NRF1/TFAM signaling pathway. Therefore, crocin might be considered as a potential therapeutic medicine to ameliorate cognitive impairment under acute highaltitude hypoxia.

\section{Data Availability}

The data used to support the findings of this study are available from the corresponding author upon request.

\section{Conflicts of Interest}

The authors declare no conflict of interest. 


\section{Authors' Contributions}

Xiaoyan Zhang, Dianxiang Lu, and Zhanqiang Li were responsible for the study concept and design. Xiaoyan Zhang, Xianjun Zhang, and Zhancui Dang performed the experiments. Zhancui Dang and Xianjun Zhang participated in data and statistical analyses. Xiaoyan Zhang, Shanshan Su, and Dianxiang $\mathrm{Lu}$ wrote the article and prepared figures. All authors read and approved the final manuscript.

\section{Acknowledgments}

This work was supported by grants from the National Natural Science Foundation of China (No. 81260685), the Basic Research Project from Qinghai Science and Technology Department of China (2016-ZJ-708), and the Youth Research Fund team project of Medical College of Qinghai University (2018-kyt-3).

\section{References}

[1] S. I. Harik, W. D. Lust, S. C. Jones, K. L. Lauro, S. Pundik, and J. C. LaManna, "Brain glucose metabolism in hypobaric hypoxia," Journal of applied physiology, vol. 79, no. 1, pp. 136140, 1995.

[2] C. Davis and P. Hackett, "Advances in the Prevention and Treatment of High Altitude Illness," Emergency Medicine Clinics of North America, vol. 35, no. 2, pp. 241-260, 2017.

[3] A. D. Titus, B. S. Shankaranarayana Rao, H. N. Harsha et al., "Hypobaric hypoxia-induced dendritic atrophy of hippocampal neurons is associated with cognitive impairment in adult rats," Neuroscience, vol. 145, no. 1, pp. 265-278, 2007.

[4] P. Maiti, S. B. Singh, B. Mallick, S. Muthuraju, and G. Ilavazhagan, "High altitude memory impairment is due to neuronal apoptosis in hippocampus, cortex and striatum," Journal of Chemical Neuroanatomy, vol. 36, no. 3-4, pp. 227238, 2008.

[5] A. Arya, A. Gangwar, S. K. Singh et al., "Cerium oxide nanoparticles promote neurogenesis and abrogate hypoxiainduced memory impairment through AMPK-PKC-CBP signaling cascade," International Journal of Nanomedicine, vol. 11, pp. 1159-1173, 2016.

[6] P. Liu, D. Zou, L. Yi et al., "Quercetin ameliorates hypobaric hypoxia-induced memory impairment through mitochondrial and neuron function adaptation via the PGC- $1 \alpha$ pathway," Restorative Neurology and Neuroscience, vol. 33, no. 2, pp. 143-157, 2015.

[7] H. Lin, C. P. Chang, H. J. Lin, M. T. Lin, and C. C. Tsai, "Attenuating brain edema, hippocampal oxidative stress, and cognitive dysfunction in rats using hyperbaric oxygen preconditioning during simulated high-altitude exposure," The journal of trauma and acute care surgery, vol. 72, no. 5, pp. 1220-1227, 2012.

[8] S. Irarrazaval, C. Allard, J. Campodonico et al., "Oxidative Stress in Acute Hypobaric Hypoxia," High Altitude Medicine \& Biology, vol. 18, no. 2, pp. 128-134, 2017.

[9] W.-H. Li, Y.-X. Li, and J. Ren, "High altitude hypoxia on brain ultrastructure of rats and Hsp70 expression changes," British Journal of Neurosurgery, vol. 33, no. 2, pp. 192-195, 2019.

[10] F. Yang, L. Zhou, J. Song et al., "Liver CEBP $\beta$ Modulates the Metabolism and Mediates the Motility for Hypoxia-Induced
Central Fatigue in Mice," Frontiers in Physiology, vol. 10, p. 243, 2019.

[11] B. L. Tang, "Sirt1 and the Mitochondria," Molecules and Cells, vol. 39, no. 2, pp. 87-95, 2016.

[12] J. Tang, L. Lu, Y. Liu et al., "Quercetin improve ischemia/reperfusion-induced cardiomyocyte apoptosis in vitro and in vivo study via SIRT1/PGC-1 $\alpha$ signaling," Journal of Cellular Biochemistry, vol. 120, no. 6, pp. 9747-9757, 2018.

[13] L. Tian, W. Cao, R. Yue et al., "Pretreatment with Tilianin improves mitochondrial energy metabolism and oxidative stress in rats with myocardial ischemia/reperfusion injury via AMPK/SIRT1/PGC-1 alpha signaling pathway," Journal of Pharmacological Sciences, vol. 139, no. 4, pp. 352-360, 2019.

[14] R. S. Khan, Z. Fonseca-Kelly, C. Callinan, L. Zuo, M. M. Sachdeva, and K. S. Shindler, "SIRT1 activating compounds reduce oxidative stress and prevent cell death in neuronal cells," Frontiers in Cellular Neuroscience, vol. 6, p. 63, 2012.

[15] V. H. Nieto Estrada, D. Molano Franco, R. D. Medina, A. G. Gonzalez Garay, A. J. Martí-Carvajal, and I. Arevalo-Rodriguez, "Interventions for preventing high altitude illness: part 1. Commonly-used classes of drugs," Cochrane Database System Reviews, vol. 6, article CD009761, 2017.

[16] R. M. Salama, G. A. Abdel-Latif, S. S. Abbas, H. M. El Magdoub, and M. F. Schaalan, "Neuroprotective effect of crocin against rotenone-induced Parkinson's disease in rats: interplay between PI3K/Akt/mTOR signaling pathway and enhanced expression of miRNA-7 and miRNA-221," Neuropharmacology, vol. 164, p. 107900, 2019.

[17] Z. Duan, H. Li, X. Qi et al., "Crocin attenuation of neurological deficits in a mouse model of intracerebral hemorrhage," Brain Research Bulletin, vol. 150, pp. 186-195, 2019.

[18] S. V. Rao, P. Hemalatha, S. Yetish, M. Muralidhara, and P. S. Rajini, "Prophylactic neuroprotective propensity of Crocin, a carotenoid against rotenone induced neurotoxicity in mice: behavioural and biochemical evidence," Metabolic Brain Disease, vol. 34, no. 5, pp. 1341-1353, 2019.

[19] X. Y. Zhang, X. J. Zhang, J. Xv et al., "Crocin attenuates acute hypobaric hypoxia-induced cognitive deficits of rats," European Journal of Pharmacology, vol. 818, pp. 300-305, 2018.

[20] R. Morris, "Developments of a water-maze procedure for studying spatial learning in the rat," Journal of Neuroscience Methods, vol. 11, no. 1, pp. 47-60, 1984.

[21] O. Mykhailenko, V. Kovalyov, O. Goryacha, L. Ivanauskas, and V. Georgiyants, "Biologically active compounds and pharmacological activities of species of the genus Crocus: A review," Phytochemistry, vol. 162, pp. 56-89, 2019.

[22] M. Pagani, D. Salmaso, G. G. Sidiras et al., "Impact of acute hypobaric hypoxia on blood flow distribution in brain," Acta physiologica, vol. 202, no. 2, pp. 203-209, 2011.

[23] C. E. Turner, S. L. Barker-Collo, C. J. Connell, and N. Gant, "Acute hypoxic gas breathing severely impairs cognition and task learning in humans," Physiology \& Behavior, vol. 142, pp. 104-110, 2015.

[24] L. Mohammadzadeh, K. Abnous, B. M. Razavi, and H. Hosseinzadeh, "Crocin-protected malathion-induced spatial memory deficits by inhibiting TAU protein hyperphosphorylation and antiapoptotic effects," Nutritional Neuroscience, vol. 23, pp. 221-236, 2019. 
[25] M. Ahmadi, Z. Rajaei, M. A. Hadjzadeh, H. Nemati, and M. Hosseini, "Crocin improves spatial learning and memory deficits in the Morris water maze via attenuating cortical oxidative damage in diabetic rats," Neuroscience Letters, vol. 642, pp. 1-6, 2017.

[26] S. N. Meydani, D. Wu, M. S. Santos, and M. G. Hayek, “Antioxidants and immune response in aged persons: overview of present evidence," The American Journal of Clinical Nutrition, vol. 62, no. 6, pp. 1462S-1476S, 1995.

[27] M. SaiRam, S. K. Sharma, P. Dipti et al., "Effect of hypobaric hypoxia on immune function in albino rats," International Journal of Biometeorology, vol. 42, no. 1, pp. 55-59, 1998.

[28] O. M. Vikbladh, M. R. Meager, J. King et al., "Hippocampal Contributions to Model-Based Planning and Spatial Memory," Neuron, vol. 102, no. 3, pp. 683-693.e4, 2019.

[29] P. Guo, H. Luo, Y. Fan, Y. Luo, and Q. Zhou, "Establishment and evaluation of an experimental animal model of high altitude cerebral edema," Neuroscience Letters, vol. 547, pp. 82$86,2013$.

[30] D. Sharma, K. K. Barhwal, S. N. Biswal et al., "Hypoxia-mediated alteration in cholesterol oxidation and raft dynamics regulates BDNF signalling and neurodegeneration in hippocampus," Journal of Neurochemistry, vol. 148, no. 2, pp. 238-251, 2019.

[31] N. S. X. Ren, M. Ji, E. J. Tokar et al., "Haploinsufficiency of SIRT1 enhances glutamine metabolism and promotes cancer development," Current biology, vol. 27, no. 4, pp. 483-494, 2017.

[32] R. Yacoub, K. Lee, and J. C. He, "The role of SIRT1 in diabetic kidney disease," Frontiers in Endocrinology, vol. 5, p. 166, 2014.

[33] M. P. Mattson, K. Moehl, N. Ghena, M. Schmaedick, and A. Cheng, "Intermittent metabolic switching, neuroplasticity and brain health," Nature Reviews Neuroscience, vol. 19, no. 2, pp. 63-80, 2018.

[34] D. Yan, C. Jin, Y. Cao et al., "Effects of on Long-Term Memory in Rats and on SIRT1 Mediating the Transcription of CREB-Dependent Gene in Hippocampus," Basic \& Clinical Pharmacology \& Toxicology, vol. 121, no. 4, pp. 342352, 2017.

[35] D. Albani, L. Polito, and G. Forloni, "Sirtuins as novel targets for Alzheimer's disease and other neurodegenerative disorders: experimental and genetic evidence," Journal of Alzheimer's Disease, vol. 19, no. 1, pp. 11-26, 2010.

[36] L. Jia, J. Wang, H. Cao, X. Zhang, W. Rong, and Z. Xu, “Activation of PGC- $1 \alpha$ and Mitochondrial Biogenesis Protects Against Prenatal Hypoxic-ischemic Brain Injury," Neuroscience, vol. 432, pp. 63-72, 2020.

[37] R. C. Scarpulla, R. B. Vega, and D. P. Kelly, "Transcriptional integration of mitochondrial biogenesis," Trends in Endocrinology and Metabolism: TEM, vol. 23, no. 9, pp. 459-466, 2012.

[38] M. S. Gilardini Montani, R. Santarelli, M. Granato et al., "EBV reduces autophagy, intracellular ROS and mitochondria to impair monocyte survival and differentiation," Autophagy, vol. 15, no. 4, pp. 652-667, 2018.

[39] T. Valero, "Mitochondrial biogenesis: pharmacological approaches," Current Pharmaceutical Design, vol. 20, no. 35, pp. 5507-5509, 2014.

[40] M. B. Hock and A. Kralli, "Transcriptional control of mitochondrial biogenesis and function," Annual Review of Physiology, vol. 71, no. 1, pp. 177-203, 2009.
[41] P. Wareski, A. Vaarmann, V. Choubey et al., "PGC-1\{alpha\} and PGC-1\{beta\} regulate mitochondrial density in neurons," The Journal of Biological Chemistry, vol. 284, no. 32, pp. 21379-21385, 2009.

[42] L. Cheng, B. Li, X. Chen et al., "CTRP9 induces mitochondrial biogenesis and protects high glucose-induced endothelial oxidative damage via AdipoR1 -SIRT1- PGC- $1 \alpha$ activation," Biochemical and Biophysical Research Communications, vol. 477, no. 4, pp. 685-691, 2016.

[43] T. Zhang, Y. Chi, Y. Ren, C. Du, Y. Shi, and Y. Li, "Resveratrol Reduces Oxidative Stress and Apoptosis in via Sir2-Related Enzymes, (SIRT1)/Peroxisome Proliferator-Activated Receptor $\gamma$ Co-Activator $1 \alpha$ (PGC-1 $\alpha$ ) Axis," Medical Science Monitor, vol. 25, pp. 1220-1231, 2019.

[44] M. Yang, J. Zhang, X. Jin, C. Li, G. Zhou, and J. Feng, "NRF1enhanced miR-4458 alleviates cardiac hypertrophy through releasing TTP-inhibited TFAM," In Vitro Cellular \& Developmental Biology Animal, vol. 56, no. 2, pp. 120-128, 2020.

[45] K. Chandrasekaran, M. Anjaneyulu, J. Choi et al., "Role of mitochondria in diabetic peripheral neuropathy: Influencing the $\mathrm{NAD}^{+}$-dependent SIRT1-PGC- $1 \alpha$-TFAM pathway," International Review of Neurobiology, vol. 145, pp. 177-209, 2019.

[46] F.-H. Li, H.-T. Yu, L. Xiao, and Y.-Y. Liu, "Response of BAX, Bcl-2 Proteins, and SIRT1/PGC- $1 \alpha$ mRNA Expression to 8Week Treadmill Running in the Aging Rat Skeletal Muscle," Advances in Experimental Medicine and Biology, vol. 923, pp. 283-289, 2016.

[47] F. Vazquez, J. H. Lim, H. Chim et al., "PGC1 $\alpha$ Expression Defines a Subset of Human Melanoma Tumors with Increased Mitochondrial Capacity and Resistance to Oxidative Stress," Cancer Cell, vol. 23, no. 3, pp. 287-301, 2013.

[48] K. Bhawe and D. Roy, "Interplay between NRF1, E2F4 and MYC transcription factors regulating common target genes contributes to cancer development and progression," Cellular Oncology, vol. 41, no. 5, pp. 465-484, 2018.

[49] J. St-Pierre, S. Drori, M. Uldry et al., "Suppression of reactive oxygen species and neurodegeneration by the PGC-1 transcriptional coactivators," Cell, vol. 127, no. 2, pp. 397-408, 2006.

[50] Q. Ye, L. Ye, X. Xu et al., "Epigallocatechin-3-gallate suppresses 1-methyl-4-phenyl-pyridine-induced oxidative stress in PC12 cells via the SIRT1/PGC- $1 \alpha$ signaling pathway," BMC Complementary and Alternative Medicine, vol. 12, no. 1, p. 82, 2012.

[51] Y. Zhang, Y. Li, Q. Feng, M. Shao, F. Yuan, and F. Liu, "Polydatin attenuates cadmium-induced oxidative stress via stimulating SOD activity and regulating mitochondrial function in _Musca domestica_ larvae," Chemosphere, vol. 248, p. 126009, 2020.

[52] H.-R. Kim, S. J. Won, C. Fabian, M.-G. Kang, M. Szardenings, and M.-G. Shin, "Mitochondrial DNA aberrations and pathophysiological implications in hematopoietic diseases, chronic inflammatory diseases, and cancers," Annals of Laboratory Medicine, vol. 35, no. 1, 2015.

[53] P. Maiti, S. B. Singh, A. K. Sharma, S. Muthuraju, P. K. Banerjee, and G. Ilavazhagan, "Hypobaric hypoxia induces oxidative stress in rat brain," Neurochemistry International, vol. 49, no. 8, pp. 709-716, 2006.

[54] S. Heidari, S. Mehri, and H. Hosseinzadeh, "Memory enhancement and protective effects of crocin against D-galactose aging model in the hippocampus of Wistar rats," Iranian Journal of Basic Medical Sciences, vol. 20, no. 11, pp. 1250-1259, 2017. 
[55] S. Shahidani, Z. Rajaei, and H. Alaei, "Pretreatment with crocin along with treadmill exercise ameliorates motor and memory deficits in hemiparkinsonian rats by anti-inflammatory and antioxidant mechanisms," Metabolic Brain Disease, vol. 34, no. 2, pp. 459-468, 2019.

[56] T. Ochiai, S. Ohno, S. Soeda, H. Tanaka, Y. Shoyama, and H. Shimeno, "Crocin prevents the death of rat pheochromyctoma (PC-12) cells by its antioxidant effects stronger than those of $\alpha$-tocopherol," Neuroscience Letters, vol. 362, no. 1, pp. 61-64, 2004.

[57] S. A. Dorri, H. Hosseinzadeh, K. Abnous, F. V. Hasani, R. Y. Robati, and B. M. Razavi, "Involvement of brain-derived neurotrophic factor (BDNF) on malathion induced depressivelike behavior in subacute exposure and protective effects of crocin," Iranian Journal of Basic Medical Sciences, vol. 18, no. 10, pp. 958-966, 2015.

[58] P. Yao, Y. Li, Y. Yang, S. Yu, and Y. Chen, "Triptolide Improves Cognitive Dysfunction in Rats with Vascular Dementia by Activating the SIRT1/PGC- $1 \alpha$ Signaling Pathway," Neurochemical Research, vol. 44, no. 8, pp. 1977-1985, 2019.

[59] M. Nasehi, S. Torabinejad, M. Hashemi, S. Vaseghi, and M.R. Zarrindast, "Effect of cholestasis and NeuroAid treatment on the expression of Bax, Bcl-2, Pgc- $1 \alpha$ and Tfam genes involved in apoptosis and mitochondrial biogenesis in the striatum of male rats," Metabolic Brain Disease, vol. 35, no. 1, pp. 183-192, 2020.

[60] S. K. Hota, K. Barhwal, S. B. Singh, and G. Ilavazhagan, "Chronic hypobaric hypoxia induced apoptosis in CA1 region of hippocampus: a possible role of NMDAR mediated $\mathrm{p} 75^{\mathrm{NTR}}$ upregulation," Experimental Neurology, vol. 212, no. 1, pp. 513, 2008.

[61] K. B. Hota, S. K. Hota, R. B. Srivastava, and S. B. Singh, "Neuroglobin regulates hypoxic response of neuronal cells through Hif- $1 \alpha$ - and Nrf2-mediated mechanism," Journal of cerebral blood flow and metabolism, vol. 32, no. 6, pp. 1046-1060, 2012.

[62] C. Lin, C. J. Wu, I. H. Wei et al., "Chronic treadmill running protects hippocampal neurons from hypobaric hypoxiainduced apoptosis in rats," Neuroscience, vol. 231, pp. 216224, 2013.

[63] L. Zhou, S. I. Wang, Y. J. Moon et al., "Overexpression of SIRT1 prevents hypoxia-induced apoptosis in osteoblast cells," Molecular Medicine Reports, vol. 16, no. 3, pp. 2969-2975, 2017. 